\title{
评 述＼cjkstart甚长波红外光电探测材料和器件专题
}

\section{阻挡杂质带甚长波红外探测器}

潘昌翊 ${ }^{1,2,3}$, 牟浩 ${ }^{1}$, 张祎 ${ }^{1,3}$, 殷子薇 ${ }^{1,3}$, 姚尧 ${ }^{1}$, 邓惠勇 ${ }^{*}$, 吴惠桢 ${ }^{4}$, 戴宁 ${ }^{1,2,3,5^{*}}$

1. 中国科学院上海技术物理研究所红外物理国家重点实验室, 上海 200083;

2. 上海科技大学物质科学与技术学院, 上海 201210;

3. 中国科学院大学, 北京 100049 ;

4. 浙江大学物理系和硅材料国家重点实验室, 杭州 310027;

5. 中国科学院大学杭州高等研究院, 杭州 310024

*联系人, 邓惠勇, E-mail: hydeng@mail.sitp.ac.cn; 戴宁, E-mail: ndai@mail.sitp.ac.cn

收稿日期: 2020-08-07; 接受日期: 2020-10-20; 网络出版日期: 2021-01-12

国家自然科学基金(编号: 11933006)、国家重点基础研究发展计划(编号: 2016YFB0402405, 2016YFA0202200)和中国科学院前沿重点研究(编 号: QYZDJ-SSW-SLH018)资助项目

摘要＼cjkstart阻挡杂质带(Blocked Impurity Band, BIB)探测器是从杂质带光电导(Impurity Band Conduction, IBC)探测器 发展而来, 利用杂质能级上的电子跃迁, 可以探测光子能量远小于半导体禁带宽度的低能光子. BIB探测器的探测波长 主要由祄底和掺杂杂质决定, 可以覆盖5-300 $\mu \mathrm{m}$ 波段范围. 得益于探测波长长、探测率高和抗辐射性好等优点, BIB 探测器自问世以来就一直被大量研究, 广泛应用于各种大型天文基红外探测平台上. 目前, Si基BIB探测器发展迅 速, 但是 $\mathrm{Ge}$ 基和 $\mathrm{GaAs}$ 基BIB探测器的发展相对缓慢. BIB探测器对于推动天文和相关科学的发展具有不可替代的 作用, 欧美等发达国家在BIB探测器上的科研投入巨大. 随着我国经济和科技的发展, 我国科研人员急切盼望国产 BIB探测器可以早日投入使用。本文主要针对BIB探测器物理模型、制备方法、测试手段和国内外发展现状等方 面展开讨论, 探究BIB探测器的主要工作机制以及器件研发的关键技术, 帮助相关研究人员快速了解BIB探测器.

关键词阻挡杂质带, BIB, 红外探测, 天文探测

PACS: 05.45.-a, 45.50.Jf, 45.50.Pk, 95.55.Pe, 96.12.De

\section{1 引言}

1979年，美国罗克韦尔国际公司的研究人员Petroff和Stapelbroek ${ }^{[1]}$ 在杂质带光电导(Impurity Band Conduction，IBC)探测器结构的基础上引入一层本征 的阻挡层，提出阻挡杂质带(Blocked Impurity Band, BIB)探测器结构. BIB探测器和IBC探测器都是利用杂
质能级吸收光子，探测波长由杂质在祄底中的电离激 活能决定 ${ }^{[2-5]}$. 由于电离激活能远小于半导体材料的禁 带宽度, 所以BIB探测器和IBC探测器可以比相应的本 征半导体探测器探测波长更长的红外信号. 杂质在半 导体中的电离激活能主要由杂质种类和基底材料决 定, $\mathrm{Si}$ 基杂质带探测器对应的探测波长在 5- $40 \mu \mathrm{m}, \mathrm{Ge}$ 基杂质带探测器的探测波长在40-200 $\mu \mathrm{m}, \mathrm{GaAs}$ 基杂

引用格式: 潘昌翊, 牟浩, 张神, 等. 阻挡杂质带甚长波红外探测器. 中国科学: 物理学 力学 天文学, 2021, 51: 027303

Pan C Y, Mou H, Zhang Y, et al. Blocked impurity band very long wavelength infrared detector (in Chinese). Sci Sin-Phys Mech Astron, 2021, 51: 027303, doi: 10.1360/SSPMA-2020-0309 
质带探测器可将探测波长拓展至 $300 \mu \mathrm{m}^{[6-16]}$. 图1列出 了常用红外天文探测器的探测波长范围和工作温 度 ${ }^{[17]}$, 杂质带电导型红外探测器主要应用在 $5 \mu \mathrm{m}$ 以上 的长波波段. 与 IBC器件相比, $\mathrm{BIB}$ 探测器有其特有的 优点. 首先, 由于 BIB结构器件可以比 $I B C$ 结构器件具 有更高的杂质浓度, 所以 BIB 探测器比IBC探测器具有 更宽的探测波长范围. 其次, 由于阻挡层的阻挡作用, BIB探测器的暗电流通常比IBC探测器小, 这对于探测 微弱辐射信号非常有益. 此外, 更高的杂质浓度使得红 外吸收能力更强, BIB探测器的器件体积可以比 $I B C$ 器 件更小而具有同样的光子吸收能力。这使BIB探测器 的抗辐照性能大幅提高, 对器件的空间应用极为有利. 上述一系列的优点使 $\mathrm{BIB}$ 器件结构被提出以来, 就受 到了各国研究人员的重视, 被大量地研究与应用.

1980年, Petroff和Stapelbroek成功研制出单元硅掺 砷(Si:As) BIB探测器, 为红外探测器的研究和应用开 辟了新的途径. 1984年, Kleihans率先对焦平面型BIB 器件展开研究, 两年后Stetson等人 ${ }^{[18]}$ 也成功研制出 BIB焦平面型器件, 使得BIB 器件的成像性能大幅提 升. 1999年, Hogue和Guptill等人研制了 Si:Ga BIB探测 器, 工作温度提升到了 14 K. 2005年, Reichertz等人 ${ }^{[19]}$ 曾用液相外延方法研制过GaAs:S BIB探测器, 但是器 件响应性能较差. 2006年, 加州大学伯克利分校的Beeman等人 ${ }^{[20]}$ 采用离子注入方法制备了 Ge:B BIB探测器, 开创了 BIB器件制备的新途径. 2008年, Hogue等人 ${ }^{[21]}$ 为了进一步提高吸收层的掺杂浓度提出了F Far-Infrared-Extended BIB (FIREBIB) 器件模型, 将Si:As探测器 的探测波长延伸到了 $50 \mu \mathrm{m}$ 以上. 2016 年，日本名古屋 大学和东京大学的Hanaoka等人 ${ }^{[22]}$ 采用表面键合技术, 将高掺杂 $\mathrm{Ge}$ 片与高纯Ge片键合到一起, 获得了杂质浓 度突变的Ge:Ga BIB器件. 表1列出了Raytheon和DRS Technologies两家公司研制的BIB焦平面探测器的主要 性能指标 ${ }^{[23]}$.

从材料体系来看, $\mathrm{Si}$ 基 $\mathrm{BIB}$ 探测器的发展较为快 速, Ge基和 GaAs基BIB探测器的发展相对缓慢. 这不

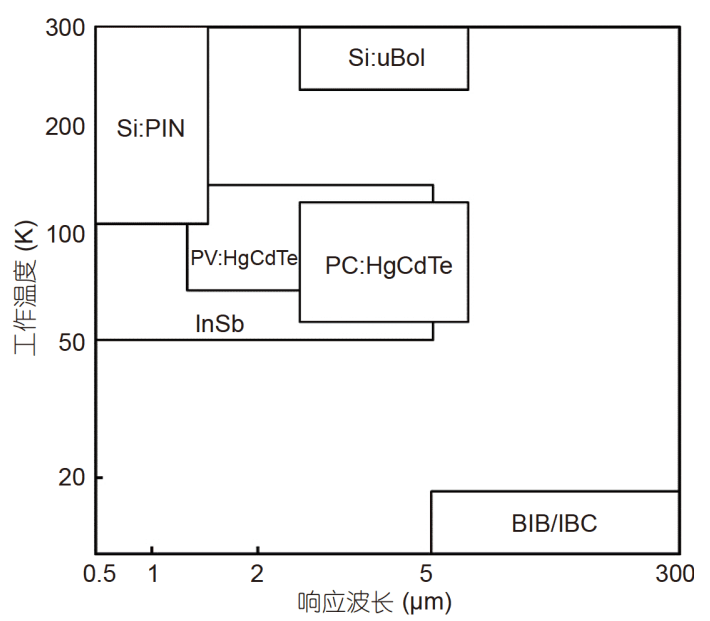

图 1 常用红外天文探测器探测波长范围和工作温度 ${ }^{[17]}$. 图 片来自文献[17], 并获得授权

Figure 1 The detection wavelength and working temperature of common infrared astronomical detectors [17]. Reprinted with permission.

仅得益于 $\mathrm{Si}$ 基材料配套的半导体工艺相对完善，而且 还与 $\mathrm{Si}$ 基 $\mathrm{BIB}$ 器件测试条件较易满足有关. 尽管 $\mathrm{Ge}$ 基 和GaAs基BIB探测器的研制难度大，但是其可探测的 波长比 $\mathrm{Si}$ 基 $\mathrm{BIB}$ 探测器更长, 因此是 $\mathrm{Si}$ 基 $\mathrm{BIB}$ 探测器所 不能替代的, 应坚持对 $\mathrm{Ge}$ 基和 $\mathrm{GaAs}$ 基 $\mathrm{BIB}$ 探测器的研 究. 从研究地区来看, 美国、日本和西欧一些国家在 BIB探测器方面开展的研究较多, 中国在这方面的研 究相对滞后, 在一定程度上制约了中国红外天文学的 发展. 本文拟简单讨论BIB探测器的物理模型、研制 方法、测试手段和国内外发展状况等, 分析发展BIB 探测器所需要的关键技术.

\section{BIB探测器物理模型}

BIB结构探测器主要由三个功能区组成：简并高 掺杂电极层、掺杂吸收层和本征阻挡层, 图2所示为 $\mathrm{N}$ 型BIB探测器的结构简图和能带简图 ${ }^{[24]}$. BIB探测器从 $\mathrm{IBC}$ 探测器发展而来, 在原本IBC结构的基础上增加了 一层高纯本征层. 当半导体中杂质的掺杂浓度增加到

表 1 主流 Si:As BIB焦平面探测器性能指标 ${ }^{[23]}$

Table 1 Key performance indicators of advanced Si:As BIB infrared focal plane arrays [23]

\begin{tabular}{cccccccc}
\hline 探测器 & 像元数 & 探测波长 $(\mu \mathrm{m})$ & 像元尺寸 $(\mu \mathrm{m})$ & 工作温度 $(\mathrm{K})$ & 暗电流 $\left(\mathrm{e}^{-}\right)$ & 读出噪声 $\mathrm{e}^{-} \mathrm{RMS}$ & 量子效率 $(\%)$ \\
\hline Si:As (DRS WISE) & $1024 \times 1024$ & $5-28$ & 18 & 7.8 & $<5$ & 42 & $>70$ \\
Si:As (Raytheon JWST) & $1024 \times 1024$ & $5-28$ & 25 & 6.7 & 1 & 10 & $>70$ \\
\hline
\end{tabular}




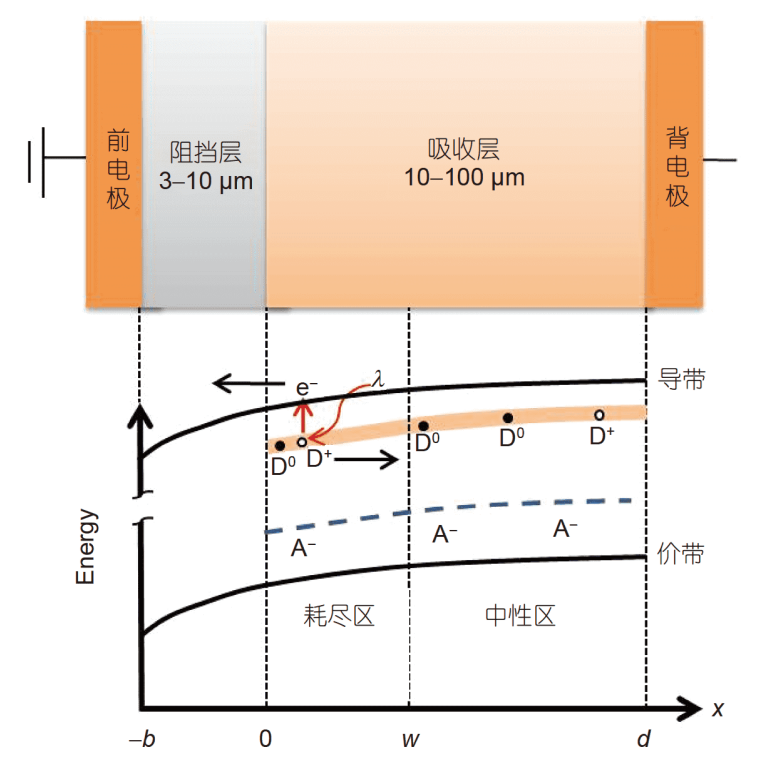

图 2 (网络版彩图) N型BIB探测器的结构简图和正常工作 模式下能带简图 $\left(\mathrm{D}^{0}\right.$ 表示中性施主杂质， $\mathrm{D}^{+}$表示电离施主杂 质, $\mathrm{A}^{-}$表示电离受主杂质)

Figure 2 (Color online) Schematic diagram and energy band structure of N-type devices on conventional operation mode $\left(\mathrm{D}^{0}\right.$ represents neutral donor impurities, $\mathrm{D}^{+}$represents ionized donor impurities, and $\mathrm{A}^{-}$ represents ionized acceptor impurities).

一定量时，相邻杂质原子价电子的波函数会发生交叠， 原本分散的杂质能级将拓宽成杂质能带. 电荷可以通 过杂质能带传输从而形成电流, 导致器件暗电流激增, 这严重制约了 $\mathrm{IBC}$ 器件的掺杂浓度. 而BIB探测器的本 征层内掺杂浓度很低, 不会形成杂质能带, 通过杂质能 带传输的暗电流将被阻挡，这也是本征层被称为阻挡 层的原因.

在N型BIB探测器的吸收层中, 除了主动掺杂的施 主杂质以外, 还有少量补偿受主杂质. 因为施主能级远 高于受主能级, 所以即使在极低温下, 施主杂质上的电 子也会离化而占据受主杂质能级. 假设所有的受主杂 质都可以获得电子而电离, 则吸收层内电离施主杂质 的浓度就等于少量受主杂质的浓度. 由于BIB探测中 施主浓度较高, 施主杂质的电子波函数相互交叠, 电 子可以在中性施主杂质和电离施主杂质之间自由运 动. 这种自由运动可以等效看成电离施主杂质沿相反 方向的运动, 即“空穴”运动. 受主杂质浓度较低, 相邻 杂质距离较远, 价电子的波函数不能发生交叠, 因此电 离受主杂质的电子不能自由运动 ${ }^{[25,26]}$. 在外加电场的 驱动下，吸收层内的电离施主杂质所贡献的电子向正
电极端运动(图2中向左方向), 可以看成电离施主杂质 向负电极端移动.

当在BIB探测器前电极端施加一个正偏压 $V$ 时(正 常工作模式), 器件内部形成电场, 电压和电场满 足 ${ }^{[27,28]}$

$V+V_{b i}=\int_{-b}^{d} E(x) \mathrm{d} x$,

式中 $V_{b i}$ 为器件内建电场, $b$ 为阻挡层宽度, $d$ 为吸收层 宽度. $V_{b i}$ 与外加偏压 $V$ 相比很小, 通常可以忽略不计. 正常工作模式下，电离施主将向背电极端移动，在吸 收层与阻挡层的边界处, 将会形成电离施主耗尽区域, 称为耗尽区, 宽度记为 $w$. 耗尽区域带负电, 净电荷密 度由器件吸收层内的补偿受主杂质浓度 $N_{a}$ 决定. 吸收 层内, 耗尽区以外的区域称为中性区, 中性区的场强 $E$ 很小, 为了方便计算, 将其近似为 0 , 即当 $w<x<d$ 时, $E(x)=0$. 在理想的阻挡层内, 没有净电荷存在.

由高斯定理, 可以精确分析器件内部的电场分布 情况:

$\frac{\mathrm{d} E}{\mathrm{~d} x}=\frac{\rho}{\varepsilon \varepsilon_{0}}= \begin{cases}0, & -b<x<0, \\ -\frac{q N a}{\varepsilon \varepsilon_{0}}, & 0<x<w,\end{cases}$

其中 $x$ 表示BIB探测器中的位置坐标, $\rho$ 为空间净电荷 密度, $\varepsilon_{0}$ 和 $\varepsilon$ 分别为真空介电常数和材料的相对介电常 数, $q$ 为电子电量. 将边界条件 $E(w)=0$ 代入式(2)计算可 得电场强度分布:

$E= \begin{cases}\frac{q w N a}{\varepsilon \varepsilon_{0}}, & -b<x<0, \\ -\frac{q N a}{\varepsilon \varepsilon_{0}}(x-w), & 0<x<w .\end{cases}$

进一步将电场强度的计算结果带入式(1)得

$V=\int_{-b}^{0} \frac{q w N a}{\varepsilon \varepsilon_{0}} \mathrm{~d} x+\int_{o}^{w}-\frac{q N a}{\varepsilon \varepsilon_{0}}(x-w) \mathrm{d} x$.

解式(4)可得耗尽层宽度为

$w=\sqrt{\frac{2 \varepsilon \varepsilon_{0} V}{q N a}+b^{2}}-b$.

当能量比杂质电离激活能大的光子辐射照射到 $\mathrm{BIB}$ 器件上时, 吸收层内杂质能级上的电子吸收光子 后跃迁到导带, 成为可以自由迁移运动的载流子. 在 耗尽层以内产生的光生载流子在电场的驱动下, 向电 源正极迁移, 形成光电流. 在耗尽层以外的吸收区, 由 
于电场强度很小，光生载流子无法高效地向电极端迁 移, 被复合掉的概率很大, 因此对光电流贡献很小. 为 了提高器件的光响应电流, 应该对器件结构进行特别 的优化设计, 以获得尽可能宽的耗尽层. 从式(5)可以 看出，耗尽层宽度主要受外加偏压、阻挡层宽度和受 主杂质浓度影响. 外加偏压越大，耗尽层宽度就越大． 然而, 外加偏压过高可能会导致器件内部电场过大, 使 得器件暗电流增大, 不利于微弱信号的探测. 阻挡层宽 度越小, 耗尽区越宽, 但是当耗尽区宽度减小到一定程 度后阻挡作用将减弱或失效. 另外, 吸收层的补偿受主 掺杂浓度越高, 耗尽区越窄. 所以, 获得尽可能宽的耗 尽区往往通过降低补偿掺杂受主浓度来实现. 在 $\mathrm{Si}$ 基 BIB器件外延生长过程中, 补偿杂质浓度通常控制在 $10^{14} \mathrm{~cm}^{-3}$ 以内. 在(3)和(5)式中设定 $N_{a}=10^{14} \mathrm{~cm}^{-3}$ 和 $b=5 \mu \mathrm{m}$, 计算得到的BIB器件在不同偏压下的电场强 度分布情况如图3(a)所示. 可见, 电场强度在阻挡层内 取得最大值, 并保持不变, 在吸收层内的电场强度相对 较小. 设定 $b=5 \mu \mathrm{m}, N_{a}$ 分别取值 $10^{13}, 10^{14}$ 和 $10^{15} \mathrm{~cm}^{-3}$, 由 式(5)可计算得到阻挡层宽度随外加偏压的变化关系, 结果如图3(b)所示. 所以，随着偏压的增加，耗尽层宽 度明显增加. 补偿掺杂浓度增高, 耗尽层宽度减小. 当 补偿掺杂浓度增加到 $10^{15} \mathrm{~cm}^{-3}$ 时, 耗尽层宽度只有纳 米级, 这将严重影响器件的有效光吸收.

照射到器件表面的电磁辐射, 并不一定能被器件 全部吸收．考虑到电磁波在器件内部的多次反射，则 光生载流子产生率为 ${ }^{[29,30]}$

$$
G(x)=\frac{\Phi \alpha\left(1-R_{1}\right)\left(\mathrm{e}^{-\alpha x}+2 R_{2} \mathrm{e}^{-2 \alpha d} \mathrm{e}^{-\alpha x}\right)}{1-R_{1} R_{2} \mathrm{e}^{-2 \alpha d}},
$$

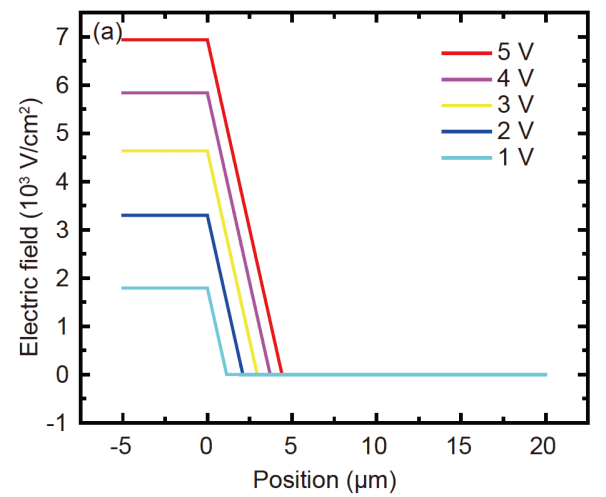

其中, $\Phi$ 表示入射到器件表面的光子通量, $R_{1}, R_{2}$ 分别为 器件前、后表面的反射率, $\alpha$ 为材料的吸收系数, $d$ 为材 料厚度. 在器件耗尽层内, 稳态时的电离施主和电子传 输电流的连续性方程为 ${ }^{[31]}$ (以下讨论仅限于正常工作 模式)

$-\frac{\mathrm{d} J_{p}}{\mathrm{~d} x}+\xi(x) J_{n}(x)+q G(x)=0$,

$\frac{\mathrm{d} J_{n}}{\mathrm{~d} x}+\xi(x) J_{n}(x)+q G(x)=0$,

$J_{p}, J_{n}$ 分别表示电离施主和电子传输电流, $\xi(x)$ 表示电子 碰撞电离系数. 在耗尽层内, 光激发产生的电子和电离 施主对在电场作用下快速分离，其复合的概率很小. BIB探测器工作在深低温环境下，热激发载流子很少. 电离施主在杂质能带上的迁移率较小，因此碰撞电离 效应很弱．在上述连续性方程中忽略了载流子复合、 热激发载流子和电离施主碰撞电离的影响.

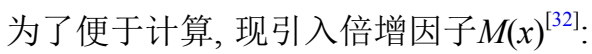

$M(x)=\mathrm{e} \int_{0}^{x} \xi\left(x^{\prime}\right) \mathrm{d} x^{\prime}$.

$M(x)$ 有两个显著的特点, 即 $\frac{\mathrm{d} M(x)}{\mathrm{d} x}=\xi(x) M(x)$ 和 $M(0)=1$. 将(7)和(8)式分别乘以 $M(x)$ 得

$\left[\frac{\mathrm{d} J_{p}}{\mathrm{~d} x}+\xi(x) J_{p}(x)\right] \cdot M(x)=[\xi(x) J(x)+q G(x)] \cdot M(x)$,

$\left[\frac{\mathrm{d} J_{n}}{\mathrm{~d} x}+\xi(x) J_{n}(x)\right] \cdot M(x)=q G(x) M(x)$.

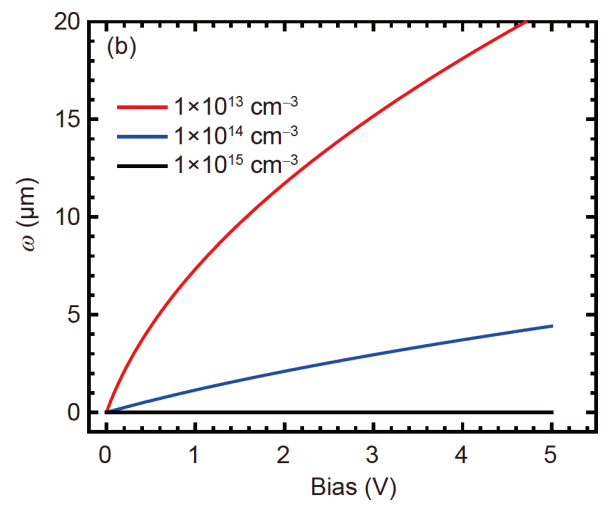

图 3 (网络版彩图) N型BIB探测器在正常工作模式下的电场分布(a)和耗尽区宽度随偏压变化关系(b)

Figure 3 (Color online) Electric field distribution (a) and dependence of depletion layer width on bias (b) in N-type BIB detectors on conventional operation mode. 
将式(10)由 0 积分到 $x$, 将式(11)由 $x$ 积分到 $w$ 得

$M(x) \cdot J_{p}(x)=J_{p}(0)+\int_{0}^{x}\left[\xi\left(x^{\prime}\right) J\left(x^{\prime}\right)+q G\left(x^{\prime}\right)\right] \cdot M\left(x^{\prime}\right) \mathrm{d} x^{\prime}$,

$M(x) \cdot J_{n}(x)=M(w) J_{n}(x)+q \int_{x}^{w} G\left(x^{\prime}\right) M\left(x^{\prime}\right) \mathrm{d} x^{\prime}$.

将式(12)与(13)相加, 可得总电流密度

$J=J_{p}(0)+M(w) J_{n}(w)+q \int_{0}^{w} G(x) M(x) \mathrm{d} x$,

其中 $J_{p}(0)$ 为阻挡层与吸收层界面处的空穴电流, 也就 是从前电极注入的空穴电流. 由于阻挡层的阻挡作用, 小偏压下, $J_{p}(0)$ 的值很小. $J_{n}(w)$ 为耗尽区和中性区边 界处, 在中性区一个扩散长度内向耗尽区注入的扩散 电流. 因为这部分电流在整个耗尽区内传输时, 一直 会倍增电离, 所以要乘上 $M(w)$. 大偏压下, $J_{n}(w)$ 还包 括从后电极注入的电子电流. 由于扩散长度与耗尽区 长度比起来很小, 所以小偏压下, 器件的光电流主要 由耗尽区内的光激发载流子贡献. 光电流的理论计算 结果进一步证明了耗尽区的重要性.

通常研究人员认为BIB探测器具有单向性, 只关 注BIB探测器在正常工作模式下的工作特性. 美国海 军研究生院的 $\operatorname{Garcia}^{[33]}$ 通过理论分析, 认为器件在反 常工作模式(前电极端接负极)下也可以很好地工作, 如图4所示. 浙江大学的Zhu等人 ${ }^{[34,35]}$ 通过实验验证了 反常工作模式的可行性. 他们认为，低温下吸收层内 杂质带上的电离施主相当于空穴，在反常工作模式下 吸收层和 $\mathrm{N}$ 型背电极之间构成了类似于PN结的结构. 由于背电极层的掺杂浓度很高，而吸收层内电离施主 的浓度很低(近似等于补偿受主浓度), 所以形成的耗 尽区几乎全部落在吸收层内. 与正常工作模式不同的 是，反常工作模式下的耗尽区在吸收层和背电极层的 界面附近.

采用与正常工作模式类似的方法, 可得电场强度 与耗尽区宽度(假设吸收层未被完全耗尽)为

$E=-\frac{q N_{a}}{\varepsilon \varepsilon_{0}}(x-w), 0 \leq x \leq w$,

$w=\sqrt{\frac{2 \varepsilon \varepsilon_{0} V}{q N_{a}}}$.

同式(5)类似, $w$ 正比于 $\sqrt{v / N_{a}}$. 从式(15)可以看出, 在吸收层与背电极层界面处, 电场强度取得最大值, 而

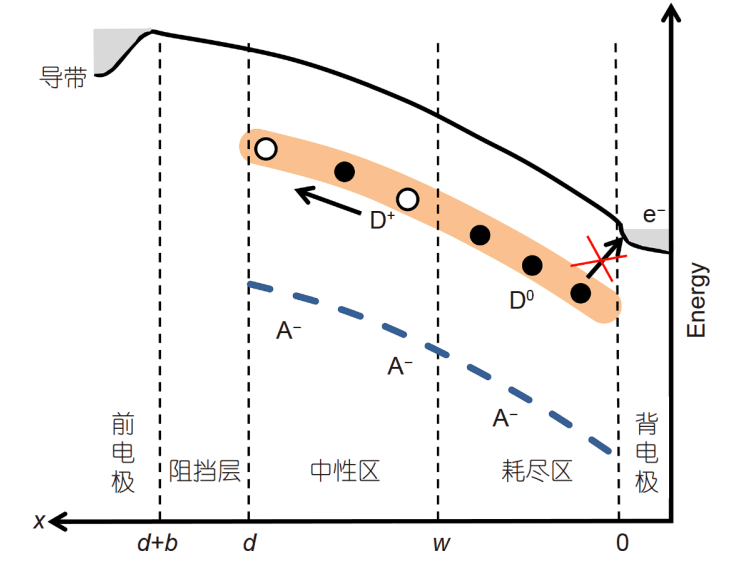

图 4 (网络版彩图) N型BIB探测器反常工作模式下能带 简图

Figure 4 (Color online) Band structure of N-type devices on alternative operation mode.

在阻挡层内, 电场强度最小. 从式(16)可以看出, 反向 工作模式下, 耗尽区的宽度只由补偿掺杂浓度和外加 偏压决定，而与阻挡层宽度无关. 设定吸收层宽度 $d=$ $20 \mu \mathrm{m}$, 阻挡层宽度 $b=5 \mu \mathrm{m}$, 补偿掺杂浓度 $N_{a}=$ $10^{14} \mathrm{~cm}^{-3}$, 由(15)和(16)式计算BIB器件在不同反向工 作电压下的电场分布, 如图5(a)所示. 中性区和阻挡层 内电场强度很小, 外加偏压几乎全部落在耗尽区内, 这 有利于光生载流子的迁移运动. 设定 $N_{a}$ 分别取值 $10^{13}$, $10^{14}$ 和 $10^{15} \mathrm{~cm}^{-3}$ 时, 耗尽区宽度随电压的变化关系如图 5(b)所示. 耗尽区宽度随偏压和补偿掺杂浓度的变化 趋势与正常工作模式一样. 值得注意的是, 在相同偏 压和相同的补偿杂质浓度下, 反向工作模式下的耗尽 层更宽.

然而, 真实的器件结构与理想的器件结构往往会 有偏差, 导致真实的器件光电特性与理想情况下的计 算结果有偏差. Haegel等人 ${ }^{[36,37]}$ 曾研究过阻挡层和吸 收层内的杂质浓度分布偏离理想情况时的电场强度分 布. 除了光电流以外, BIB探测器中还存在很小的暗电 流. 暗电流的来源主要有: 简并掺杂电极层的注入电 流、强电场下碰撞电离电流和杂质能级上的热激发噪 声电流. 为了减小电极层的注入电流, Martin等人 ${ }^{[38,39]}$ 曾在BIB结构器件上设计了异质结势垒和肖特基势垒, 效果很显著. 为了减小碰撞电离导致的暗电流, $\mathrm{BIB}$ 器 件通常工作在较低的偏压下. Si基BIB探测器通常工作 在几伏电压以内, 而 $\mathrm{Ge}$ 基BIB探测器则可以在几十毫 伏的电压下工作. 为了减小热激发噪声电流, BIB器件 
对工作温度的要求非常高. 文献报道的 $\mathrm{Si}$ 基 $\mathrm{BIB}$ 探测器 通常的工作温度为 $4-15 \mathrm{~K}$, 而 $\mathrm{Ge}$ 基 $\mathrm{BIB}$ 的工作温度在 液氦以下.

\section{BIB探测器的制备方法}

通常可以采用外延生长和离子注入两种方法制备 BIB探测器，前者采用外延生长的方法生长器件吸收 层和阻挡层，而后者采用离子注入的方法制备器件的 掺杂吸收层. 外延型器件属于垂直结构，不同的功能 区位于不同的外延层内．根据电磁辐射照射的方式, 垂直型器件又可以分为背照式和前照式两种结构，如 图6所示 ${ }^{[17]}$. 背照式结构BIB探测器工作时, 红外辐射 先透过高纯祄底然后再照射到器件吸收区域，而前照
式BIB结构让红外辐射先穿过阻挡层然后再入射到器 件吸收区域. 这两种不同的器件结构所涉及的器件制 备工艺也不同. 为了减少入射辐射进入吸收层之前被 缺陷和杂质吸收，背照式结构需要采用质量很高的高 纯基底. 而前照式结构则需要简并掺杂的祄底，以保 证光生载流子可以高效地迁移到电极端. 背照式结构 的优点是, 可以使器件工作区域紧贴低温杜瓦的热承, 有利于获得更低和更稳定的工作温度. 前照式结构的 优点是吸收区离电磁辐射入射面近，相比背照式减小 了辐射信号的传播损失. 离子注入型器件的所有功能 区都在材料很浅的表面层, 属于平面型结构器件, 如 图7所示. 离子注入掺杂的深度主要由离子注入能 量、注入杂质质量和祄底种类决定, 通常在 $1 \mu \mathrm{m}$ 以内, 限制了器件对入射辐射的有效吸收. 奥地利林茨大学
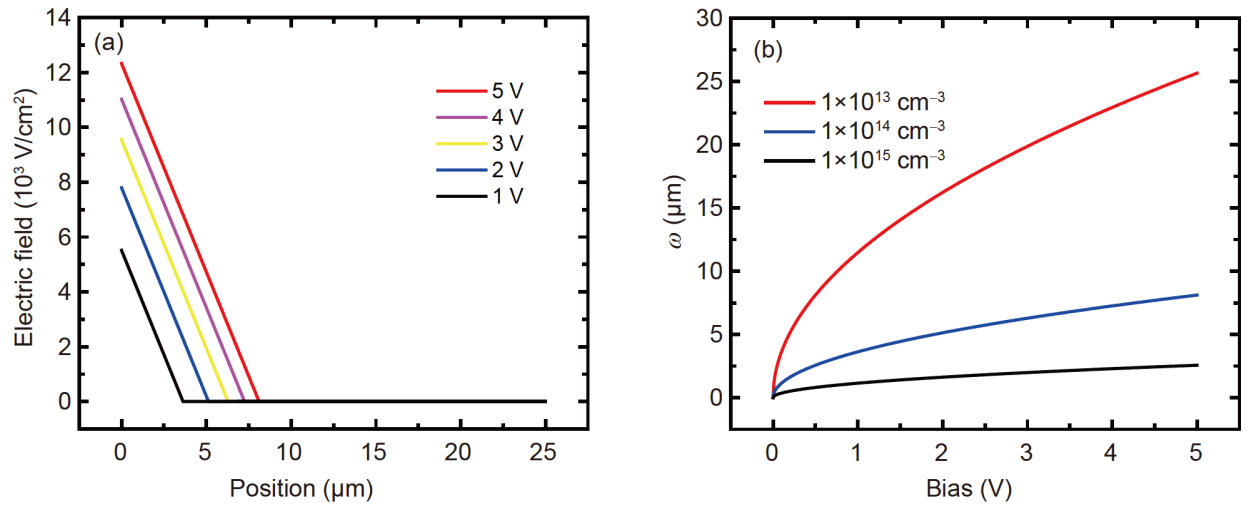

图 5 (网络版彩图) N型BIB探测器在反常工作模式下的电场分布(a)和耗尽区宽度随偏压变化图(b)

Figure 5 (Color online) Electric field distribution (a) and dependence of depletion layer width on bias (b) in N-type BIB detectors on alternative operation mode.

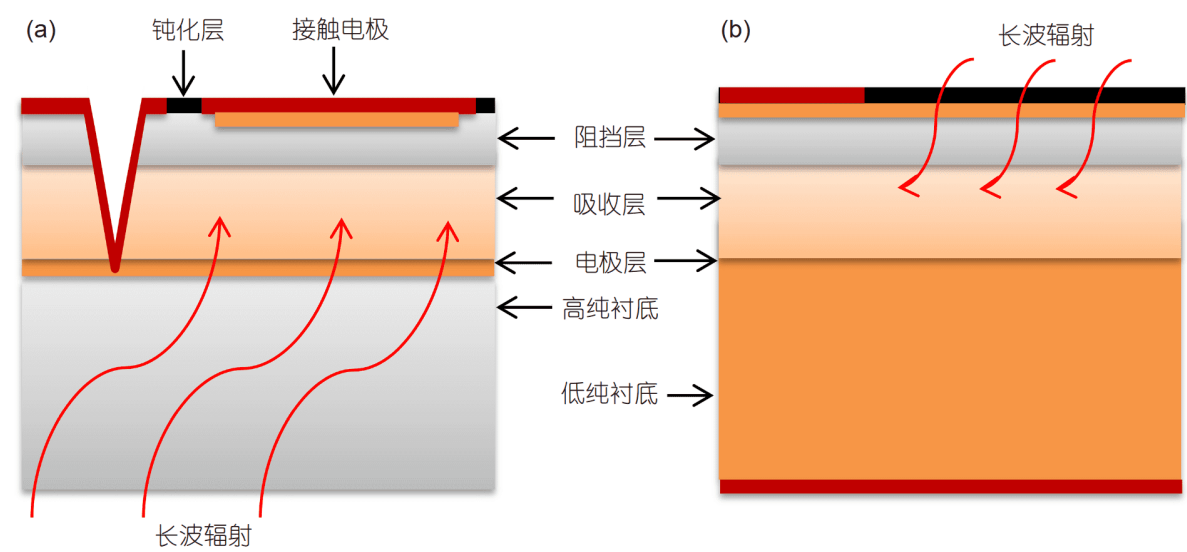

图 6 (网络版彩图)背照式(a)和前照式(b) BIB探测器结构图 ${ }^{[17]}$. 图片来自文献[17], 并获得授权

Figure 6 (Color online) Structure of back- (a) and front-illuminated (b) BIB detector [17]. Reprinted with permission. 


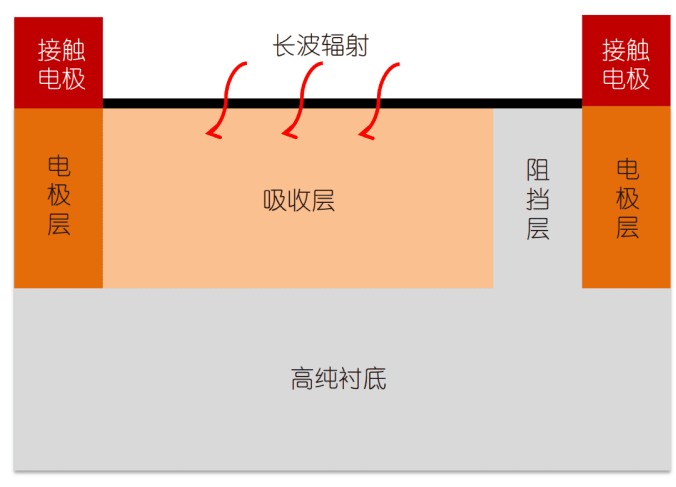

图 7 (网络版彩图)平面型BIB探测器结构图

Figure 7 (Color online) Structure of planar BIB detector.

的Rauter等人 ${ }^{[40]}$ 曾用超高能离子注入机, 制备了 $10 \mu \mathrm{m}$ 厚的垂直结构BIB探测器. 离子注入的优点在于能精 确控制杂质的浓度、均匀性和掺杂区域等. 目前，应 用较多的还是外延生长型BIB器件, 离子注入的方法 主要用于制备 $\mathrm{Ge}$ 基和 $\mathrm{GaAs}$ 基BIB探测器.

下面以背照式结构为例，简单介绍制备外延型 BIB探测器的工艺流程. 如图8所示, 第一步先在高纯 祄底上外延生长背电极层、吸收层和阻挡层. 外延电 极层的厚度一般为几百纳米, 吸收层的厚度为几十微 米，阻挡层的厚度为几微米. 第二步采用离子注入掺 杂方法制备前电极层. 由于外延生长工艺比较复杂, 所以常采用离子注入的方法制备前电极层. 第三步用 湿法腐蚀的方法开 $\mathrm{V}$ 形槽. 开 $\mathrm{V}$ 形槽之前, 需要在表面 先沉积一层 $\mathrm{SiO}_{2}$ 或 $\mathrm{SiN}_{x}$ 等针化层作为腐蚀掩蔽层. 然 后通过光刻和干法刻蚀工艺将 $\mathrm{V}$ 形槽区域的掩蔽层去 掉. 最后再选用合适的腐蚀液, 一直腐蚀到背电极层. 由于腐蚀液腐蚀材料时的各向异性，所以腐蚀出的槽 会呈现成 V字形. 第四步沉积金属电极. 沉积的金属要 根据祄底和掺杂杂质类型选定, 以形成欧姆接触.

外延生长型BIB探测器对生长工艺要求很高: (1) 阻挡层的掺杂浓度足够低, 以保证较好的暗电流阻挡
作用; (2) 吸收层的补偿杂质浓度足够低, 以获得较宽 的耗尽区; (3) 吸收层的主要掺杂杂质浓度精确可控且 均匀, 以避免产生局部漏电流; (4) 阻挡层和外延层厚 度可控, 以满足BIB探测器的设计需求. 在BIB探测器 材料的生长过程中, 通常需要对生长设备的腔体进行 特殊清洁, 以提高洁净度, 减小背景杂质浓度的影响. 有些腔体本身就是一种杂质源, 所以用于BIB探测器 外延生长的设备往往需要特制的腔体. 生长完吸收层 或者电极层以后，腔体中就会有残留杂质，会影响阻 挡层的纯净度. 生长过程是高温工艺, 吸收层中杂质 很容易向阻挡层扩散. 此外, 商业上获得的源材料, 通 常也会由于纯净度不够而需要再次提纯. 常用的外延 生长方法有液相外延(Liquid Phase Epitaxy, LPE)、气 相外延(Vapor Phase Epitaxy, VPE)、金属有机化合物 化学气相沉积(Metal Organic Chemical Vapor Deposition, MOCVD)和分子束外延(Molecular Beam Epitaxy, $\mathrm{MBE})$ 等 ${ }^{[41-43]}$. 其中液相外延和气相外延对设备要求 低、器件制备成本易于控制. 加州大学伯克利分校的 Bandaru等人 ${ }^{[44]}$ 和Cardozo 等人 ${ }^{[45]}$ 曾用这些方法成功研 制了 Ge基和 GaAs基BIB探测器. 金属有机化合物化学 气相沉积有助于大规模批量化生产, 但是杂质浓度的 精确控制较难. 杭州电子科技大学的张耘 ${ }^{[46]}$ 曾经尝试 过用此方法研制 $\mathrm{GaAs}$ 基BIB探测器. 分子束外延法可 以精确控制掺杂浓度和晶体生长质量, 但是由于生长 速度缓慢, 很难获得BIB探测器所需要的外延厚度.

离子注入型BIB器件避免了复杂的外延生长工艺, 制备过程主要包括: 清洗、光刻、离子注入、退火、 针化和金属化等, 如图9所示. 清洗是一个非常重要的 流程, 衬底表面的洁净度对后续工艺会产生很大影响. 对于长时间放置的祄底需要采用RCA技术彻底清洗, 每次清洗需要使用丙酮、异丙醇和去离子水等. 清洗 完之后，需要在显微镜下检查是否清洗干净，以及表 面是否有划痕. 光刻工艺用来在材料表面形成特定的

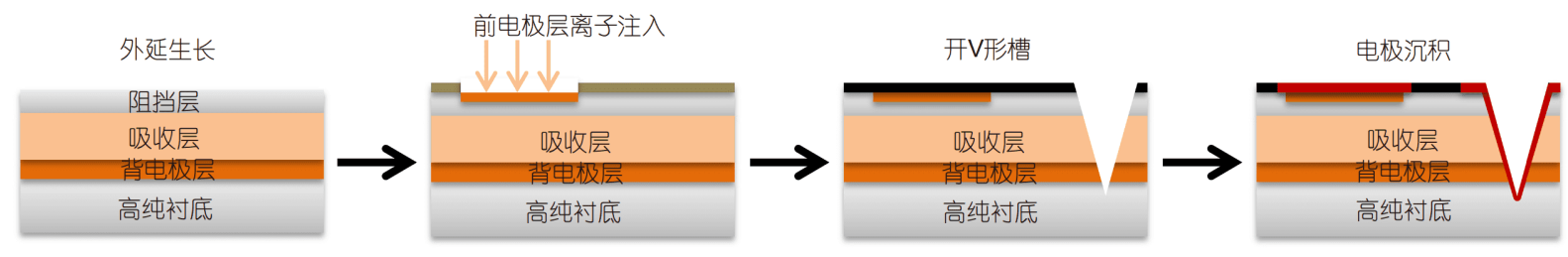

图 8 (网络版彩图)外延型BIB探测器工艺流程图

Figure 8 (Color online) Flow chart of epitaxial BIB detector. 


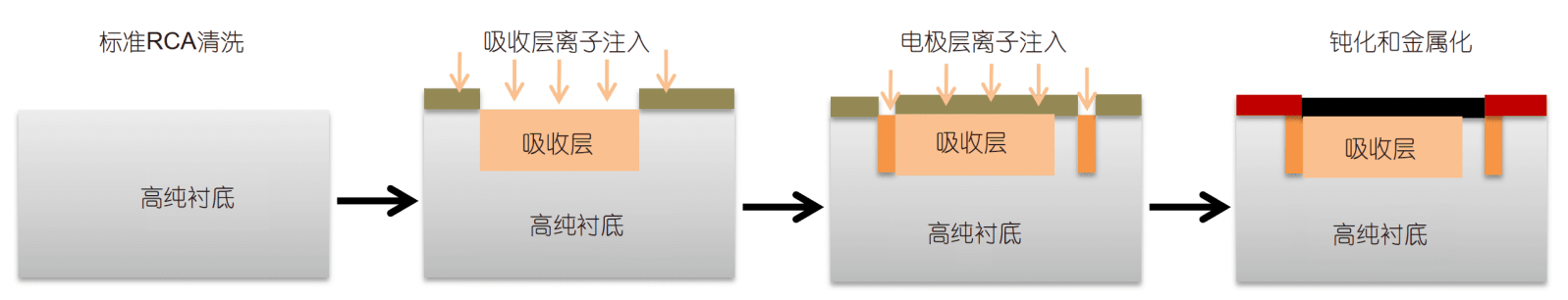

图 9 (网络版彩图)离子注入型器件工艺流程图

Figure 9 (Color online) Flow chart of ion-implanted devices.

图形，以进行局部区域的离子注入掺杂和金属电极沉 积. 离子注入之前的光刻工艺需要使用厚度较厚的光 刻胶(通常在 $3 \mu \mathrm{m}$ 以上), 作为离子注入时的掩蔽层. 离 子注入时, 由于高能杂质离子的轰击, 光刻胶很容易碳 化, 给后续清洗带来困难. 对于 $\mathrm{Si}$ 基祄底, 通常使用 piranha溶液(浓硫酸和双氧水混合液)去除碳化的光刻 胶. 由于 $\mathrm{Ge}$ 会被浓硫酸和双氧水腐蚀，所以一般不用 piranha溶液清洗Ge基祄底. 离子注入时, 为了使注入 深度尽可能深，通常选用质量较轻的离子. 提高注入 能量也可以使注入深度更深，但是注入能量越高，造 成的晶格损伤也越严重. 为了使掺杂杂质浓度均匀, 需要进行不同能量和剂量离子的多次注入. 借助Silivaco和SRIM等软件可以对离子注入过程进行模拟，通 过SIMS 实验可以检测注入深度. 通常使用快速退火技 术来修复晶格损伤和激活注入的杂质离子. 快速退火 的时间很短，可以减少吸收层和阻挡层界面处杂质的 扩散, 有利于获得杂质浓度突变的分界面. 退火操作 时, 为了防止掺杂的杂质从祄底表面析出, 需要先做 一层针化层. 同外延型器件工艺一样, 最后需要选取 特定的金属制作欧姆接触电极. 离子注入方法制备 BIB器件必须使用掺杂浓度非常低的祄底材料. 一般 来说, 对于 $\mathrm{Si}$ 晶圆常温下电阻率应大于 $10000 \Omega \mathrm{m}$, 对 于 $\mathrm{Ge}$ 晶圆常温电阻率要大于 $35 \Omega \mathrm{cm}$.

\section{BIB探测器测试系统}

$\mathrm{BIB}$ 探测器的工作温度低、探测波长长, 因此相 应的器件测试条件非常苛刻. BIB探测器通常工作在 液氦温度, 测试时需要将器件封装在液氦杜瓦里. 随 着工作温度升高, 吸收层杂质能级上的电子可以通过 热激发跃迁到导带, 使器件暗电流增大, 影响器件工 作性能. 器件的工作温度与杂质电离能、杂质掺杂浓
度和阻挡层宽度有关. 杂质电离能越小、掺杂浓度越 高、阻挡层宽度越窄, 所需要的工作温度越低. BIB探 测器主要用来探测长波长红外辐射, 因此器件测试需 要长波辐射光源. 常见的长波长光源有黑体和量子级 联激光器等. 黑体属于宽光谱光源, 黑体响应测试可 以得到器件在一个很宽波段范围内平均的响应率和探 测率等信息. 为了得到器件在特定波段的响应率和探 测率, 通常需要借助傅里叶红外光谱仪获得器件的波 长响应率, 然后对黑体测试结果进行标定. 此外, 也可 以借助特定波长的滤光片, 从黑体辐射中分离出波长 范围很窄的辐射. 量子级联激光器属于单波段光源, 可以直接测试器件在单个波段的响应率和探测率 ${ }^{[47]}$. 通常情况下, $\mathrm{Si}, \mathrm{Ge}$ 和 GaAs中杂质的电离能依次减小, 所以 $\mathrm{Si}, \mathrm{Ge}$ 和 $\mathrm{GaAs}$ 基 $\mathrm{BIB}$ 探测器所需要的工作温度依 次降低, 所需要的测试光源波长依次增长.

$\mathrm{BIB}$ 探测器是宽波段响应器件, 我们既关心器件 对特定波段辐射的响应, 也关心器件在一个很宽波段 内的平均响应特性, 因此BIB器件测试通常采用黑体 作为光源. 图10为黑体测试系统布线图, 主要用到黑 体、斩波器、低温杜瓦、前置电流放大器、锁相放大 器和示波器等设备. 黑体的温度通常控制在300$1000 \mathrm{~K}, 300,500$ 和 $800 \mathrm{~K}$ 黑体辐射的峰值波长分别在 9.7, 5.8和3.6 $\mu \mathrm{m}$ 处, 处于 $\mathrm{Si}$ 基BIB探测器的探测范围之 内. 黑体的出射孔比器件吸收区域略大, 以确保黑体辐 射全完覆盖器件吸收区域. 从黑体发出的辐射, 首先经 过斩波器调制成特定频率的辐射. 然后让调制后的黑 体辐射通过红外传输窗口照射到器件表面. 对于 $\mathrm{Si}$ 基 BIB探测器可以选用KRS-5材料制作杜瓦窗口, Ge基 和GaAs基探测器则可以选用PE和金刚石材料制作杜 瓦窗口. 前置电流放大器给器件提供一个工作偏压, 并 将响应电流放大后传输到锁相放大器. 锁相放大器根 据斩波器的调制频率, 将特定频率的信号提取出来. 


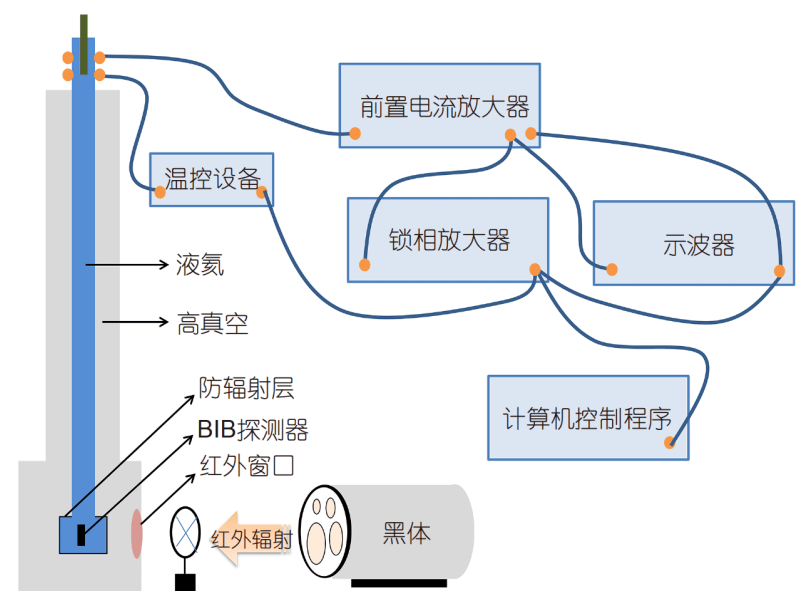

图 10 (网络版彩图) BIB探测器的黑体测试系统

Figure 10 (Color online) Blackbody test system for BIB devices.

锁相放大器的读数除以前置电流放大器的放大倍数, 就是器件的响应光电流. 锁相放大器属于高精度仪器, 量程设置不对时很容易导致仪器损坏. 因此需要先用 示波器粗略测量输出信号的大小, 然后再用锁相放大 器精确测量.

黑体响应率 $R_{\mathrm{bb}}$ 可以由下式计算得到 ${ }^{[48]}$ :

$R_{\mathrm{bb}}=\frac{\int_{0}^{+\infty} r(\lambda) M_{\mathrm{bb}}(\lambda) T_{\mathrm{r}}(\lambda) \mathrm{d} \lambda}{\int_{0}^{+\infty} M_{\mathrm{bb}}(\lambda) T_{\mathrm{r}}(\lambda) \mathrm{d} \lambda}=\frac{I_{\mathrm{ph}}}{P_{s}}$,

其中 $r(\lambda)$ 为器件在特定波段处的绝对响应率, $M_{\mathrm{bb}}(\lambda)$ 为 黑体在特定波段处的辐射出射度， $T_{\mathrm{r}}(\lambda)$ 为红外窗口透 过率, $I_{\mathrm{ph}}$ 为器件的光电流, $P_{s}$ 为单位时间内入射到器件 上的辐射能量.

黑体响应探测率可由下式估算 ${ }^{[49]}$ :

$D^{*}=\frac{R_{\mathrm{bb}}}{\sqrt{2 q I_{\mathrm{dark}}}} \sqrt{A_{d}}$,

其中 $I_{\mathrm{dark}}$ 为器件暗电流, $A_{d}$ 为器件光敏元面积.

任何温度大于 $0 \mathrm{~K}$ 的物质, 都会向外发出电磁辐 射. 通常的黑体辐射测试系统, 需要在低温杜瓦上开 设辐射窗口，这就容易受到室温背景辐射的影响。为 了模拟BIB探测器在太空中工作时的低温背景, 上海 技术物理研究所康亭亭研究员设计了一套低温黑体测 试系统. 这套系统将黑体模块集成到了低温杜瓦内部, 不需要在杜瓦外壁开设辐射窗口, 可以很好地避免室 温背景辐射的影响. 黑体模块和BIB器件之间要做好
隔热处理，避免黑体对器件工作温度造成影响. 图11 所示为20-50 K低温黑体的辐射出射度曲线, 20,30 和 $50 \mathrm{~K}$ 黑体辐射的峰值波长分别在 145,97 和 $58 \mu \mathrm{m}$ 处, 处 于 $\mathrm{Ge}$ 基和 $\mathrm{GaAs}$ 基 $\mathrm{BIB}$ 探测器的探测范围之内. 因此, 与高温黑体测试系统相比, 低温黑体测试系统不仅可 以更好地模拟BIB探测器在太空中运行时的背景辐射, 而且也更符合 $\mathrm{Ge}$ 基和 $\mathrm{GaAs}$ 基BIB探测器的测试需求.

\section{BIB探测器应用}

研制BIB探测器的目的是抑制器件暗电流，拓展 探测器的探测波长, 实现器件在长波红外天文观测和 军事防御领域的应用. 长波红外探测的重要性主要体 现在以下两个方面：(1) 宇宙中很多星系和尘埃的温 度较低(几K到几十 $\mathrm{K}$ ), 峰值辐射波长位于远红外区; (2) 可见光的穿透性差, 容易被星际尘埃阻挡, 很难超 远距离传输. BIB探测器可探测波长覆盖5-300 $\mu \mathrm{m}$, 能 很好地满足长波长探测需求. 自BIB探测器被研制成 功以来，其一直被用于各种大型天基探测平台，大大 提高了人类探测未知宇宙的能力, 促进了红外天文和 相关科学探索的实施.

1989年, 宇宙背景探测器COBE发射升空, 首次装 备了两个基于BIB结构的 $\mathrm{Si}$ Ga探测器, 用于12-25 $\mu \mathrm{m}$ 波段信号的探测. COBE卫星主要用于针对 $1 \mu \mathrm{m}-1 \mathrm{~cm}$ 波段内早期宇宙背景辐射的精确测量. 图12(a)为 COBE卫星在太空中运行时的示意图, 图12(b)为COBE 卫星在不同波段获取的天空假彩色图. 从图12(b)可以

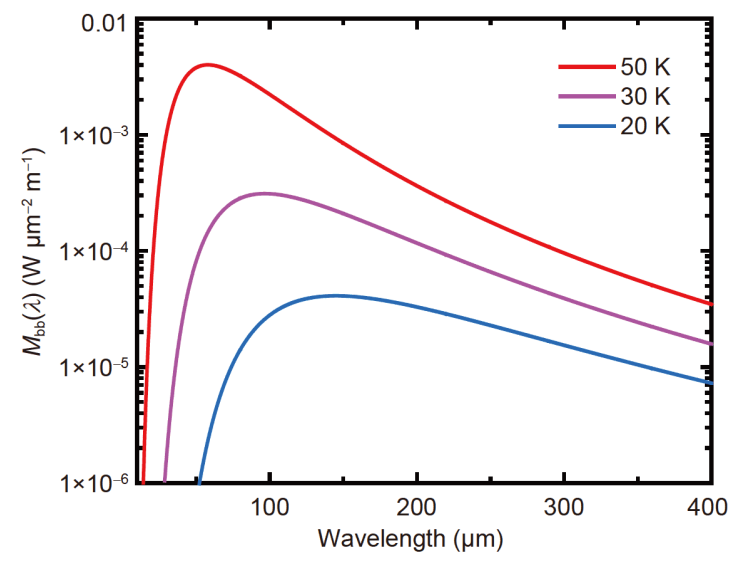

图 11 (网络版彩图) 20-50 K低温黑体辐射出射度曲线 Figure 11 (Color online) Radiation emission curve of 20-50 K low temperature blackbody. 

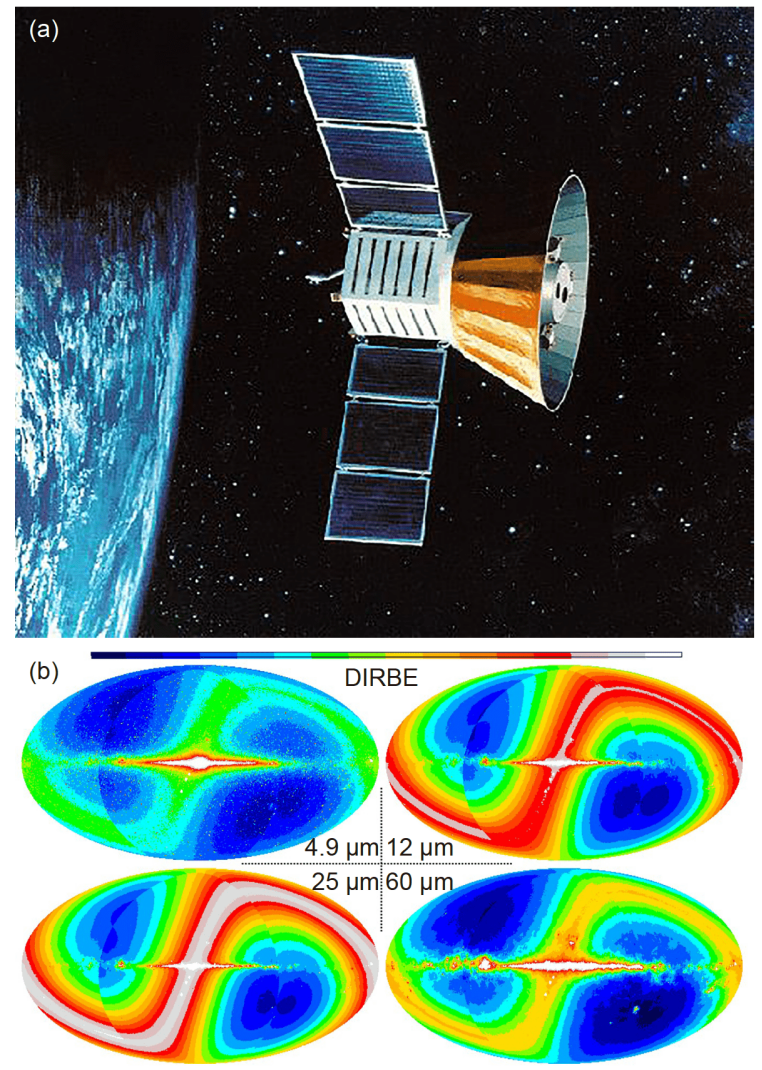

图 12 (网络版彩图) (a) COBE卫星太空运行示意图; (b) COBE卫星通过 $4.9,12,25,60 \mu \mathrm{m}$ 四个波段获得的天空假彩 色图. 数据来源于 https://science.nasa.gov/missions/cobe/和 https://lambda.gsfc.nasa.gov/product/cobe/dirbe_image.cfm

Figure 12 (Color online) (a) Schematic diagram of COBE satellite; (b) false-color image of the near-infrared sky as seen by COBE satellite at $4.9,12,25$ and $60 \mu \mathrm{m}$. The data is obtained from: https://science.nasa. gov/missions/cobe/ and https://lambda.gsfc.nasa.gov/product/cobe/dirbe_image.cfm.

看出，不同波段拍摄的图像，所包含的信息不同. COBE卫星获取的图像帮助人们加深了对早期宇宙的 更深理解，一定程度上佐证了宇宙起源的大爆炸理论. 2006年，诺贝尔物理学奖授予了COBE卫星的主要研 究者, 美国戈达德航天中心的天体物理学家Mather和 加州大学伯克利分校物理系的教授Smoot ${ }^{[50,51]}$.

2003年, Spitzer太空望远镜发射升空, 它是美国宇 航局大型轨道天文台计划中的最后一颗望远镜. 该计 划的另外三个探测平台是哈勃可见光望远镜(Hubble Space Telescope, HST)、康普顿伽马射线天文台 (Compton Gamma Ray Observatory, CGRO)和钱德拉 X射线天文台(Chandra X-ray Observatory, CXO). Spitizer主要工作在红外波段. 2017年, 科学家利用Spitizer
观测到的数据，在TRAPPIST-1恒星周围发现了 7颗大 小和地球相近且可能有液态水存在的行星 ${ }^{[52]}$. 这 7 颗 行星中有 3 颗被确定位于宜居带内, 很可能有生命存 在, 引起了科学家们的广泛关注. 图13所示为TRAPPIST-1恒星系统示意图. Spitizer携带的制冷剂最初预 计可用 2.5 年, 但是在太空极冷环境下, 实际使用了 5.5 年. 制冷剂用完后, Spitizer装备的部分探测器在所处 环境温度下继续工作, 直到2020年才完全退役.

2009年，宽视场红外探测卫星WISE投入使用。该 卫星装备了大规模BIB焦平面型探测器，探测灵敏度 和成像性能大幅提升. WISE卫星的主要目标是绘制整 个宇宙的红外波段图像，图14为WISE卫星观测到的

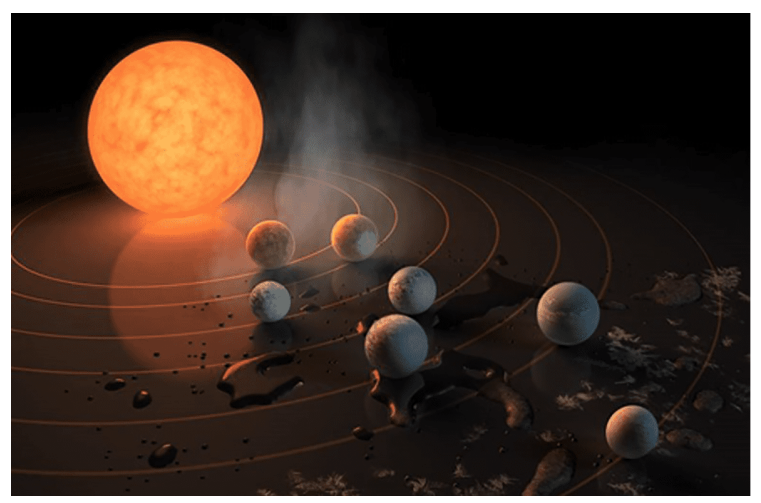

图 13 (网络版彩图) TRAPPIST-1恒星系统示意图. 数据来 源: https://www.nasa.gov/mission_pages/spitzer/images/index. html

Figure 13 (Color online) Schematic diagram of TRAPPIST-1 system. The data is obtained from https://www.nasa.gov/mission_pages/spitzer/ images/index.html.

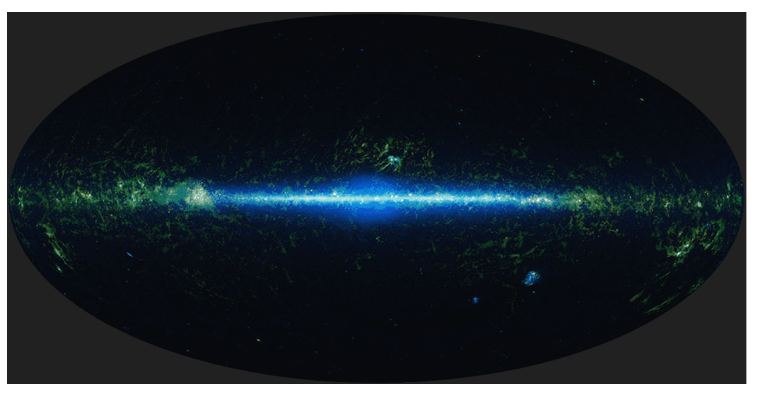

图 14 (网络版彩图) WISE绘制的宇宙全景图(3.4和4.6 $\mu \mathrm{m}$ 的光为蓝色, $12 \mu \mathrm{m}$ 的光为绿色, $22 \mu \mathrm{m}$ 的光为红色). 数据来 源: https://www.nasa.gov/feature/jpl/celebrating-10-years-ofthe-wise-spacecraft

Figure 14 (Color online) The sky as seen by WISE (3.4- and 4.6- $\mu \mathrm{m}$ light is colored blue, $12-\mu \mathrm{m}$ light is green, and $22-\mu \mathrm{m}$ light is red). The data is obtained from https://www.nasa.gov/feature/jpl/celebrating-10years-of-the-wise-spacecraft. 
3.4, 4.6, 12 和 $22 \mu \mathrm{m} 4$ 个波段的宇宙图像. WISE卫星携 带的制冷剂于2011年消耗完，此后进入短暂的休眠状 态，后于2013年被重新激活，并通过NEOWISE项目继 续运行. 虽然只有短短两年的服役期, WISE卫星却有 很多重大发现: (1) 发现了宇宙中最亮的星系, 其亮度 超过 300 万亿个太阳; (2) 帮助排除了“X”星的存在, 而 之前很多科学家认为天王星和海王星绕日轨道的不规 则是由于太阳系边缘存在“X”行星; (3) 发现了数百万 个黑洞，这些黑洞通过可见光望远镜很难观测到; (4) 发现了最冷的恒星, 其温度比人体温度更低. WISE卫 星的观测数据已被数以千计的学术论文引用.

表2列举了一些主要的天文基红外探测平台 ${ }^{[53-57]}$, 它们全部装备了 IBC 和BIB结构的探测器. 得益于半导 体技术的发展, BIB探测器已由最初的单元器件发展 到了如今的超大规模焦平面型器件. 即将发射的詹姆 斯·韦伯空间望远镜(JWST)仍然会在其长波红外成像 系统中采用BIB结构的器件. JWST望远镜已投资约 100 亿美元，将成为哈勃空间望远镜的继任者. 早期的 红外天文卫星通常使用液氦作为制冷剂，这限制了卫 星的有效工作时长. 随着制冷机技术的发展，新型天 文卫星有效工作时间不断延长. 长波红外探测对于探 索宇宙起源、发现地外生命和探寻特殊星体有着不可 替代的作用, 得到了越来越多国家的重视. 现在主流的 天文探测平台都会装备甚长波红外波段的BIB探测器. 近年来, 国内中国科学院上海技术物理研究所、浙江 大学、中国电工科技集团公司第五十研究所等单位也 开展了 $\mathrm{BIB}$ 探测器的研究, 期待着不远的将来BIB长波
红外探测器在我国的红外天文卫星上得到应用.

\section{6 总结与展望}

BIB探测器具有探测波长长、探测率高和抗辐射 性能好等优点, 非常适用于长波红外天文探测领域. 在 过去几十年间, 研究人员对BIB结构探测器开展了大 量研究, 建立了基本的暗电流和光电流的物理模型, 开发了系统的器件制备工艺. BIB探测器已经被广泛 应用到各种大型天文探测平台上，大大提高了在红外 波段的探测能力, 并取得了令人瞩目的成就. 然而, $\mathrm{BIB}$ 探测器仍然存在一些急需解决的问题, 具体表现 在以下 5 个方面.

(1) 现有的BIB器件理论模型过于理想化, 忽略了 很多物理细节, 以至于由理论模型计算得到的器件响 应曲线很难与实验测得的结果精确吻合.

(2) 目前 $\mathrm{BIB}$ 器件的应用主要局限在 $\mathrm{Si}$ 基祄底上, 而对于 $\mathrm{Ge}$ 基和 $\mathrm{GaAs}$ 基BIB探测器的应用较少, 这很大 程度上限制了 $\mathrm{BIB}$ 器件的探测波长.

(3) BIB探测器的工作温度很低, 通常在液氦以下 温度. 当天文基探测平台上携带的液氦制冷剂消耗完 后, BIB探测器的探测性能会大大降低, 这严重限制了 探测平台的有效工作时间.

(4) 常见的BIB测试系统, 都会受到室温背景辐射 的影响, 不能真实地模拟 BIB 器件在太空中的工作环 境. 实验室得到的BIB探测器工作性能并不能真实反 应探测器在太空中运行时的性能.

表 $2 \mathrm{Si}$ 基红外天文望远镜

Table 2 Si-based astronomical infrared telescopes

\begin{tabular}{|c|c|c|c|}
\hline 卫星 & 国家/地区 & 发射时间 & 采用的 $\mathrm{Si}$ 基探测器 \\
\hline IRAS & USA/UK/Holland & 1983 & IBC Si:As (15) \\
\hline COBE & USA & 1989 & BIB Si:Ga (2) \\
\hline ISO & ESA & 1995 & $\begin{array}{c}\text { CAM: IBC Si:Ga }(32 \times 32) \\
\text { PHT: IBC Si:Ga }(1 \times 64) \\
\text { SWS: IBC Si:Ga (12), Si:Sb (2) } \\
\text { BIB Si:As (12) }\end{array}$ \\
\hline Spitzer & USA & 2003 & $\begin{array}{l}\text { IRAC: BIB Si:As }(256 \times 256) \\
\text { IRS: BIB Si:As }(128 \times 128) \\
\text { BIB Si:Sb }(128 \times 128) \\
\text { MIPS: BIB Si:As }(128 \times 128)\end{array}$ \\
\hline WISE & USA & 2009 & BIB Si:As $(1024 \times 1024)$ \\
\hline JWST & USA & to be launched & MIRI: BIBSi:As (1024×1024) \\
\hline
\end{tabular}


(5) BIB 探测器的应用主要局限在天文探测和军 事领域，而在其他领域，特别是商用领域的应用很少， 这限制了BIB探测器的推广.

针对以上存在的问题, 今后BIB探测器的研究工 作可以重点从以下 5 个方面展开.

(1) 进一步完善BIB器件物理模型, 使物理模型可 以定量地预测实验结果, 指导器件设计. 具体的方法包 括：1）考虑阻挡层的杂质影响，特别是对阻挡层内电 势和电场分布的影响；2) 考虑吸收层不均匀掺杂的影 响, 特别是吸收层与阻挡层界面区域的不均匀掺杂; 3) 考虑吸收层内中性区中的电场分布情况；4）考虑温度 对材料基本物理参数的影响，特别是液氦以下温度材 料的一些基本能带特性和载流子输运特性.

(2) 改善外延生长工艺，提高 $\mathrm{Ge}$ 和 GaAs外延生长 材料的质量, 使其满足BIB器件的设计要求. 此外, 也 可以利用离子注入方法制备BIB器件，绕开复杂的材 料生长工艺. 使用注入能量更高的离子注入设备, 解 决杂质注入深度较浅的问题.

(3) 改进BIB器件结构, 提高器件的工作温度. 可以
增加新的结构, 进一步阻挡器件暗电流, 使得温度升高 时, 器件的暗电流还保持在较低水平. 改进深低温制冷 技术, 使得装备在卫星上的制冷设备可以提供BIB器 件所需的工作温度环境.

(4) 搭设新型测试系统, 模拟器件在太空中的真实 运行环境. 可以将测试光源集成到低温杜瓦内部, 避免 在杜瓦的防辐射层和外壁上开设光学窗口.

(5) 降低BIB器件的生产成本, 提高BIB器件的生 产效率, 使其在商用领域具有一定的性价比. 针对商用 领域的需求, 设计针对性的BIB产品, 比如特定的探测 波长和工作温度等.

虽然欧美等发达国家已经将BIB探测器投入到实 际应用中, 但是我国BIB探测器方面的工作尚处于起 步阶段. 限制我国BIB探测器发展的关键技术主要是: 半导体材料外延技术、低温焦平面读出电路技术和低 温制冷技术. 为了促进我国BIB探测器的发展, 必须加 大对这些技术的研究力度. 发展BIB探测器, 不仅对我 国红外天文学的发展有重要意义, 也能有力推动我国 半导体技术的发展.

\section{参考文献}

1 Petroff M D, Stapebroek M G. Blocked impurity band detectors. USA Patent, 4568960, 1986-02-04

2 Rollin B V, Simmons E L. Long wavelength infra-red photoconductivity of silicon at low temperatures. Proc Phys Soc B, 1952, 65: 995-996

3 Burstein E, Oberly J J, Davisson J W. Infrared photoconductivity due to neutral impurities in silicon. Phys Rev, 1953, 89: 331-332

4 Rollin B V, Simmons E L. Long wavelength infra red photoconductivity of silicon at low temperatures. Proc Phys Soc B, 1953, 66: 162-168

5 Sclar N. Properties of doped silicon and germanium infrared detectors. Prog Quantum Electron, 1984, 9: 149-257

6 Jeffrey V C, Herter T, Butturini R, et al. Evalution of Si:As and Si:Sb blocked impurity band detectors for SIRTF and WISE. In: Proceedings of International Symposium on Optical Science, Engineering, and Instrumentation. San Diego: SPIE, 1995

7 Hogue H H, Atkins E, Reynolds D, et al. Updated on blocked impurity band detector technology from DRS. In: Proceedings of Detectors and Imaging Devices: Infrared, Focal Plane, Single Photon. San Diego: SPIE, 2010

8 Hogue H H, Guptill M T, Monson J C, et al. Far-infrared blocked impurity band detector development. In: Proceedings of Infrared Spaceborne Remote Sensing and Instrumentation. San Diego: SPIE, 2007

9 Haegel N M. BIB detector development for the far infrared: From Ge to GaAs. In: Proceedings of Quantum Sensing: Evolution and Revolution from Past to Future. San Jose: SPIE, 2003

10 Watson D M, Huffman J E. Germanium blocked-impurity-band far-infrared detectors. Appl Phys Lett, 1988, 52: 1602-1604

11 Reichertz L A, Beeman J W, Cardozo B L, et al. GaAs BIB photodetector development for far-infrared astronomy. In: Proceedings of Infrared Spaceborne Remote Sensing XII. San Jose: SPIE, 2004

12 Tezcan D S, Putzeys J, Munck K D, et al. Development of a Si:As blocked impurity band detector for far IR detection. In: Proceedings of Infrared Systems and Photoelectronic Technology II. San Jose: SPIE, 2007

13 Bandaru J, Beeman J W, Haller E E, et al. Growth and performance of Ge:Sb blocked impurity band (BIB) detectors. In: Proceedings of Infrared Spaceborne Remote Sensing IX. San Jose: SPIE, 2002

14 Liao K S, Li N, Wang C, et al. Extended mode in blocked impurity band detectors for terahertz radiation detection. Appl Phys Lett, 2014, 105: 
143501

15 Zhu H, Weng Z, Zhu J, et al. Surface plasmon enhanced Si-based BIB terahertz detectors. Appl Phys Lett, 2017, 111: 053505

16 Simon M S, Kwok K N. Physics of Seminconductor Devices. New Jersey: John Wiley \& Sons, 2007

17 Liao K S, Liu X H, Huang L, et al. Blocked impurity band infrared detector for astronomy (in Chinese). Sci Sin-Phys Mech Astron, 2014, 44: 360-367 [廖开升, 刘希辉, 黄亮, 等. 天文用阻挡杂质带红外探测器. 中国科学: 物理学 力学 天文学, 2014, 44: 360-367]

18 Stetson S B, Reynolds D B, Stapelbroek M G, et al. Design and performance of blocked-impurity-band detector focal plane arrays. In: Proceedings of Infrared Detector, Sensors, and Focal Plane Arrays. San Diego: SPIE, 1986

19 Reichertz L A, Cardozo B L, Beeman J W, et al. First results on GaAs blocked impurity band (BIB) structures for far-infrared detector arrays. In: Proceedings Infrared Spaceborne Remote Sensing. San Diego: SPIE, 2005

20 Beeman J W, Goyal S, Reichertz L A, et al. Ion-implanted Ge:B far-infrared blocked-impurity-band detectors. Infrared Phys Tech, 2007, 51: 6065

21 Hogue H H, Mlynczak M G, Abedin M N, et al. Far-infrared detector development for space-based Earth observation. Proc SPIE, 2008, 7082: E1-E8

22 Hanaoka M, Kaneda H, Oyabu S, et al. Development of blocked-impurity-band-type Ge detectors fabricated with the surface-activated wafer bonding method for far-infrared astronomy. J Low Temp Phys, 2016, 184: 225-230

23 Rieke G H. Infrared detector arrays for astronomy. Annu Rev Astron Astrophys, 2007, 45: 77-115

24 Bandaru J. Liquid Phase Epitaxial Growth and Characterization of Germanium Far Infrared Blocked Impurity Band Detectors. Dissertation for Doctoral Degree. Berkeley: University of California, 2001

25 Miller A, Abrahams E. Impurity conduction at low concentrations. Phys Rev, 1960, 120: 745-755

26 Mott N F, Twose W D. The theory of impurity conduction. Adv Phys, 1961, 10: 107-163

27 Zhao K H, Chen X M. Electromagnetism (in Chinese). Beijing: Higher Education Press, 2006 [赵凯华, 陈熙谋. 电磁学. 北京: 高等教育出版社, 2006]

28 Liao K S. Silicon-based Blocked Impurity Band Infrared Detectors for Space Detection (in Chinese). Dissertation for Doctoral Degree. Shanghai: Shanghai Institute of Technical Physics, Chinese Academy of Sciences, 2015 [廖开升. 硅基阻挡杂质带红外探测器研制及其光电特性研究. 博士学位论文. 上海: 中国科学院上海技术物理研究所, 2015]

29 Tang D Y, Mi Z Y. Introduction to Optoelectronic Devices (in Chinese). Shanghai: Shanghai Science and Technology Literature Press, 1989 [汤 定元, 糜正瑜. 光电器件概论. 上海: 上海科学技术文献出版社, 1989]

30 Shen X C. Semiconductor Spectrum and Optical Properties (in Chinese). Beijing: Science Press, 2001 [沈学础. 半导体光谱和光学性质. 北京: 科学出版社, 2001]

31 Szmulowicz F, Madarasz F L. Blocked impurity band detectors-An analytical model: Figures of merit. J Appl Phys, 1987, 62: 2533-2540

32 McIntyre R J. Multiplication noise in uniform avalanche diodes. IEEE Trans Electron Devices, 1966, ED-13: 164-168

33 Garcia J C. Alternate Configurations for Blocked Impurity Band Detectors. Dissertation for Doctoral Degree. Monterey: Naval Postgraduate School, 2004

34 Zhu H, Weng Z, Zhu J, et al. Comparison of photoresponse of Si-based BIB THz detectors. IEEE Trans Electron Devices, 2017, 64: 1094-1099 Zhu J, Zhu H, Xu H, et al. Ge-based mid-infrared blocked-impurity-band photodetectors. Infrared Phys Tech, 2018, 92: 13-17 Haegel N M, Jacobs J E, White A M. Modeling of steady-state field distributions in blocked impurity band detectors. Appl Phys Lett, 2000, 77 : $4389-4391$

37 Haegel N M, Samperi S A, White A M. Electric field and responsivity modeling for far-infrared blocked impurity band detectors. J Appl Phys, 2003, 93: $1305-1310$

38 Martin B G, Fathauer R W, Jones E W, et al. Blocking injected dark current in impurity-band-conduction photodetectors using a PtSi Schottky barrier. Appl Phys Lett, 1995, 67: 774-776

39 Martin B G, Lee H P, Jones E W. GaAs/AlAs/Si heterostructures for blocking dark current injection in impurity-band-conduction photodetectors. Appl Phys Lett, 1996, 68: 1250-1252

40 Rauter P, Fromherz T, Winnerl S, et al. Terahertz Si:B blocked-impurity-band detectors defined by nonepitaxial methods. Appl Phys Lett, 2008, 93: 261104

41 Wang Y B, Xu W H, You T G, et al. $\beta-\mathrm{Ga}_{2} \mathrm{O}_{3}$ MOSFETs on the Si substrate fabricated by the ion-cutting process. Sci China-Phys Mech Astron, 
2020, 63: 277311

42 Zhang Y C, Li Y F, Wang Z Z, et al. Investigation of $\beta-\mathrm{Ga}_{2} \mathrm{O}_{3}$ films and $\beta-\mathrm{Ga}_{2} \mathrm{O}_{3} / \mathrm{GaN}$ heterostructures grown by metal organic chemical vapor deposition. Sci China-Phys Mech Astron, 2020, 63: 117311

43 Li K, Yang X, Tian Y, et al. $\mathrm{Ga}_{2} \mathrm{O}_{3}$ solar-blind position-sensitive detectors. Sci China-Phys Mech Astron, 2020, 63: 117312

44 Bandaru J, Beeman J W, Haller E E. Far-infrared absorption in Sb-doped Ge epilayers near the metal-insulator transition. Appl Phys Lett, 2002, 80: $3536-3538$

45 Cardozo B L, Haller E E, Reichertz L A, et al. Far-infrared absorption in GaAs:Te liquid phase epitaxial films. Appl Phys Lett, 2003, 83: 39903992

46 Zhang Y. Research on Technology of Novel Terahertz Blocked Impurity Band Detectors (in Chinese). Dissertation for Master's Degree. Hangzhou: Hangzhou Dianzi University, 2017 [张耘. 新型太赫兹BIB探测器工艺技术研究. 硕士学位论文. 杭州: 杭州电子科技大学, 2017]

47 Cao J C. Terahertz quantum cashed lasers (in Chinese). Physics, 2006, 35: 632-636 [曹俊诚. 太赫兹量子级联激光器研究进展. 物理, 2006, 35: 632-636]

48 Rogalski A. Infrared Detectors. New York: CRC Press, 2010

49 Liu X H, Zhou X H, Li N, et al. Effects of bias and temperature on the intersubband absorption in very long wavelength GaAs/AlGaAs quantum well infrared photodetectors. J Appl Phys, 2014, 115: 124503

50 Mather J C, Cheng E S, Eplee R E J, et al. A preliminary measurement of the cosmic microwave background spectrum by the cosmic background explorer (COBE) satellite. Astrophys J, 1990, 354: L37

51 Smoot G F, Bennett C L, Kogut A, et al. Structure in the COBE differential microwave radiometer first-year maps. Astrophys J, 1992, 396: L1

52 Gillon M, Triaud A H M J, Demory B O, et al. Seven temperate terrestrial planets around the nearby ultracool dwarf star TRAPPIST-1. Nature, 2017, 542: 456-460, arXiv: 1703.01424

53 Wright E L, Eisenhardt P R M, Mainzer A K, et al. The wide-field infrared survey explorer (WISE): Mission description and initial on-orbit performance. Astron J, 2010, 140: 1868-1881, arXiv: 1008.0031

54 Werner M W. The spitzer space telescope mission. Adv Space Res, 2005, 36: 1048-1049

55 Li J X, Deng J S, Xu C, et al. Development of the infrared space astronomical observatory (in Chinese). Prog Astron, 2016, 34: 327-340 [李佳席, 邓劲松, 许春, 等. 红外空间天文发展. 天文学进展, 2016, 34: 327-340]

56 Huang C, Wang J J, Gao X, et al. Survey for foreign infrared astronomical telescopes (in Chinese). Laser Infrared, 2013, 43: 235-239 [黄晨, 王建 军, 高昕, 等. 国外红外天文望远镜发展现状. 激光与红外, 2013, 43: 235-239]

57 Love P J, Hoffman A W, Lum N A, et al. 1024×1024 Si:As IBC detector arrays for JWST MIRI. In: Proceedings of International Society for Optics and Photonics. San Diego: SPIE, 2005 


\title{
Blocked impurity band very long wavelength infrared detector
}

\author{
PAN Chang Yi ${ }^{1,2,3}$, MOU Hao ${ }^{1},{\text { ZHANG } \mathrm{Yi}^{1,3}, \text { YIN ZiWei }^{1,3}, \text { YAO Yao }}^{1}$, DENG HuiYong ${ }^{1 *}$, \\ WU HuiZhen ${ }^{4} \&$ DAI Ning ${ }^{1,2,3,5^{*}}$ \\ ${ }^{1}$ State Key Laboratory of Infrared Physics, Shanghai Institute of Technical Physics, Chinese Academy of Sciences, \\ Shanghai 200083, China; \\ ${ }^{2}$ School of Physical Science and Technology, ShanghaiTech University, Shanghai 201210, China; \\ ${ }^{3}$ University of Chinese Academy of Sciences, Beijing 100049, China; \\ ${ }^{4}$ Department of Physical and State Key Laboratory of Silicon Materials, Zhejiang University, Hangzhou 310027, China; \\ ${ }^{5}$ Hangzhou Institute for Advanced Study, University of Chinese Academy of Sciences, Hangzhou 310024, China
}

Blocked impurity band (BIB) detector is a derivative of the impurity band conduction (IBC) detector. It can detect lowenergy photons whose energy is much smaller than the band gap of the semiconductor through the electronic transition on the impurity band. The detection wavelength of the BIB detector is mainly determined by the substrate and doped impurities which can cover the range of 5-300 $\mu \mathrm{m}$. Owing to the advantages of long detection wavelength, high detection rate and good radiation resistance, BIB detectors have been extensively studied and are widely used in various large-scale astronomical infrared detection platforms. Presently, Si-based BIB detectors are developing rapidly, but the development of Ge-based and GaAs-based BIB detectors is relatively slow. BIB detectors play an irreplaceable role in promoting the development of astronomy and related sciences. Developed countries such as Europe and the United States have invested a lot in scientific research on BIB detectors. With the development of China's economy and technology, Chinese researchers hope eagerly that the domestic BIB detector can be put into use as soon as possible. Herein, in order to help the related researchers to quickly understand the BIB detector, the device physical model, preparation methods, testing methods and development status have been discussed, and the involved working mechanism and key device technology are also explored.

blocked impurity band, BIB, infrared detection, astronomical detection

PACS: 05.45.-a, 45.50.Jf, 45.50.Pk, 95.55.Pe, 96.12.De

doi: 10.1360/SSPMA-2020-0309 